\title{
Theoretical and simulated models for an irreversible Otto cycle
}

\author{
P. L. Curto-Risso, ${ }^{\text {a) }}$ A. Medina, ${ }^{\text {b) }}$ and A. Calvo Hernández ${ }^{\text {c) }}$ \\ Departamento de Física Aplicada, Universidad de Salamanca, 37008 Salamanca, Spain
}

(Received 23 June 2008; accepted 6 August 2008; published online 12 November 2008)

\begin{abstract}
We show in this work that a finite-time-thermodynamics model of an irreversible Otto cycle is suitable to reproduce performance results of a real spark ignition heat engine. In order to test our model we have developed a computer simulation including a two-zone combustion model and compared the evolution of the performance parameters of the simulated engine as functions of the rotational speed $(\omega)$ with those obtained from a simple theoretical scheme including chemical reactions. A theoretical Otto cycle with irreversibilities arising from friction, heat transfer through the cylinder walls, and internal losses properly reproduces simulation results by considering extreme temperatures and mass inside the cylinder as functions of $\omega$. Furthermore we obtain realistic values for the parameters characterizing global irreversibilities, their evolution with $\omega$, and a clearer understanding of their physical origin not always well established in theoretical models. (C) 2008 American Institute of Physics. [DOI: 10.1063/1.2986214]
\end{abstract}

\section{INTRODUCTION}

During the past years the thermodynamic analysis of real heat engines has evolved at least from two different schemes. On one hand, finite-time thermodynamics (FTT) proposes an approximation based on classical equilibrium thermodynamics. ${ }^{1-4}$ Irreversibility sources are described in terms of a reduced set of parameters in order to find realistic upper bounds for the engine performance from analytic or numerical simple expressions for efficiency, power, or any other appropriate objective function. Of course, as individual processes are not studied at a microscopic detail, the obtained results should be only considered as a first approximation. However, this knowledge is interesting because the analytic simplicity of the models allows us to put in order the relative importance of the different irreversibility factors in terms of the involved parameters and variables.

Particularly, with respect to spark ignition reciprocating heat engines several theoretical approaches were developed during the last 20 years. Mozurkewich et al. ${ }^{5}$ used optimalcontrol theory to obtain the optimal motion of the piston in the Otto cycle. Aizenbud et al. ${ }^{6}$ and Chen et al. ${ }^{7}$ studied these heat engines by using the optimal motion law of a piston fitted in a cylinder containing a gas pumped at a given heating rate. Orlov et $a l^{8}{ }^{8}$ focused their studies on the power and efficiency limits for internal combustion engines. Angulo-Brown et al. ${ }^{9}$ and Calvo Hernández et al. ${ }^{10}$ modeled the Otto cycle considering friction-loss terms. Klein ${ }^{11}$ and Chen et $a l^{7}$ studied the consequences of heat transfer on the performance of the Otto cycle and their combined effect with friction irreversibilities. Angulo-Brown et al. ${ }^{12}$ also presented an Otto cycle model that includes power losses due to a lumped friction term and an explicit combustion reaction at

\footnotetext{
a) Also at Intituto de Ingeniería Mecánica y Producción Industrial, Universidad de la República Oriental del Uruguay. Electronic mail: pcurto@fing.edu.uy.

b) Also at ETSII de Béjar, Universidad de Salamanca, 37700 Béjar, Salamanca, Spain. Electronic mail: amd385@usal.es.

${ }^{c)}$ Electronic mail: anca@usal.es.
}

the end of the adiabatic compression. Recently, Ge et al. ${ }^{13}$ studied the performance of an air-standard Otto cycle with variable specific heats of the working fluid and heat resistance and friction irreversible losses. Ozsoysal ${ }^{14}$ also studied the relationship between heat losses and fuel energy and its influence on the performance of standard Otto and Diesel cycles.

On the other hand, other kinds of approaches have been developed: the numerical or computer simulation of the cycles representing spark ignition heat engines. They are based on a detailed description of every process involved in the heat engine evolution. ${ }^{15-17}$ A set of coupled differential equations for the basic thermodynamic and mechanical variables is proposed and solved after assuming several simplifying hypotheses. ${ }^{18}$ Then performance results can be obtained and compared with experimental data. With respect to experiments on benchmark engines, simulations are cheaper and flexible because they allow us to study the influence of individual variables. An extensive review on second-law analysis applied to internal combustion engines was recently reported by Rakopoulos et al. ${ }^{18}$

Although most simulations take as starting point the air standard Otto cycle, some aspects affecting thermodynamic efficiency have been added, as those accounting for fresh mixture inside the cylinder, finite rate of combustion, ${ }^{19,20}$ heat losses, and unburned charge fraction. Also, increased emphasis is placed on the detailed chemistry to improve the understanding of emissions. The proper design of an engine and its control strategy often ends up as a trade off among its efficiency, emissions, and several practical constraints. ${ }^{21}$ Accordingly, many different engine simulation models ${ }^{16}$ have been developed and, usually, they are classified according to the number of spatial directions in which the differential formulation is retained. Multidimensional models make use of an spatial resolution to solve the set of partial differential equations. In zero-dimensional (or thermodynamical) models all variables are averaged over a finite volume and an empirical correlation is used to solve the combustion process. 
The quasidimensional models improve the zero-dimensional models with the assumption of a spherical flame front and the incorporation of two additional differential equations which describe the combustion evolution. ${ }^{22}$

The drawbacks of multidimensional models include complex implementation and solution and increased uncertainty in the modeling process. For zero-dimensional models main drawbacks are oversimplified; geometrical variance and strong dependence of the empirical formulation on the combustion process were not consideration. Quasidimensional models are limited to particular geometrical configurations and, like zero-dimensional models, sometimes oversimplify the distribution of state variables. However, the quasidimensional models have beneficial results, comparing the advantages with the added difficulties.

From the point of view of FTT the objective of this paper is to find the changes to make in simple well-known models to reproduce results from simulations, not only qualitatively but also with some numerical precision. This is important to understand the physical mechanisms of the processes occurring in a real operating engine, particularly the unavoidable irreversibility sources. Recently Hoffmann et $a .^{23}$ compared the results from a zero-dimensional simulation with a simple Novikov model (a Carnot engine with finite heat conductance and heat leak between heat reservoirs) and concluded that FTT can reproduce with some detail simulations. Along this line, our aim is to solve an Otto cycle based on an FTT model including chemical reactions in the combustion and the consideration of some parameters depending on $\omega$ (the rotational speed of the engine in $\mathrm{rad} / \mathrm{s}$ ) and to compare the results with those obtained from a computational quasidimensional model.

The paper is organized as follows. In Sec. II we briefly present the differential equations of our simulation scheme and the models considered for each of the physical processes involved in a real spark ignition engine. Sec. III is devoted to present a FTT model of an irreversible Otto cycle including chemical reactions and the main irreversibility sources. In Sec. IV numerical results from the theoretical model and the simulation are presented. In this section main parameters of the FTT model are considered constant, as in previous works, and we show that in this way theoretical model only reproduces some partial results of simulations. In Sec. V we consider minimum and maximum temperatures, mass inside the cylinder, and two irreversibility parameters as speed dependent and show how this improved FTT model is capable to reproduce simulations to a very good extent. Finally, in Sec. VI we include a discussion on the physical meaning of irreversibility parameters and summarize the main conclusions of the paper.

\section{NUMERICAL SIMULATION OF AN OTTO ENGINE CYCLE}

We consider an internal combustion reciprocating engine with each cylinder performing four strokes of its piston (two revolutions of the crankshaft). Particularly, spark ignition engines develop the following strokes:
1) Intake stroke: the piston moves from the top center (TC) to the bottom center (BC) introducing fresh mixture into the cylinder. To increase the inducted mass, usually the inlet valve opens shortly before the stroke starts and closes after it ends. Consequently, there exists an overlapping period when both valves are simultaneously open.

2) Compression stroke: both valves are closed and the mixture is compressed to a lower volume. Close to the end of this stroke, a spark initiates combustion, making pressure to increase rapidly.

3) Power stroke: starts with the piston at TC and gases in the chamber at high temperature and high pressure pushing the piston down and making the crank rotate. Much work is obtained from this stroke in comparison with the work required for compression. Before the piston reaches $\mathrm{BC}$, exhaust valve opens and pressure drops.

4) Exhaust stroke: the remaining burned gases leave the cylinder. As the piston approaches TC inlet valve opens and the cycle begins again.

Our numerical model is based upon the differential form of the first principles of thermodynamics for open systems, it takes the cylinder interior as control volume and presents a general formulation of differential equations for temperature and pressure. We assume a two-zone model for combustion, thus we distinguish between burned $(b)$ and unburned $(u)$ gases. Except during combustion, the working fluid could be considered as an adiabatic mixture of both gases. We suppose all gases as ideal with $T$ - and $p$-independent gas constant, $R_{u}$ or $R_{b}$, a constant fuel ratio, $\phi$, and except in combustion, only sensible enthalpy changes, i.e., associated with temperature variations.

A differential equation for either burned or unburned gases temperature inside the cylinder can be written, except in the combustion period (that will be considered later on), as

$$
\begin{aligned}
\frac{d T}{d t}= & \frac{1}{\left(m_{u} c_{p, u}+m_{b} c_{p, b}\right)}\left[\dot{Q}_{u}+\dot{Q}_{b}+\dot{m}_{\mathrm{in}} h_{\mathrm{in}}+\dot{m}_{\mathrm{ex}} h_{\mathrm{ex}}\right. \\
& \left.-\dot{m}_{u} h_{u}-\dot{m}_{b} h_{b}+V \frac{d p}{d t}\right] .
\end{aligned}
$$

In the above equation the term $\dot{m}_{u} h_{u}+\dot{m}_{b} h_{b}$ corresponds to enthalpy changes of the gas mixture inside the cylinder, while $\dot{m}_{\text {in }} h_{\text {in }}$ and $\dot{m}_{\text {ex }} h_{\text {ex }}$ correspond to the enthalpy changes associated with intake or exhaust processes. $\dot{m}_{\text {in }}$ and $\dot{m}_{\mathrm{ex}}$ can be positive or negative depending on the relative pressures between the cylinder interior and the intake or exhaust pressures. $\dot{Q}_{u}$ and $\dot{Q}_{b}$ corresponds, respectively, to the heat transferred for unburned or burned gases. In the processes where unburned gases inside the cylinder do not exist, $m_{u}=0$ and then $\dot{Q}_{u}=0$, and the same applies for the burned gases. So, each term for $u$ or $b$ can appear or not in the equation depending on the considered stroke. The initial values are given by the external conditions.

For pressure, with the same arguments, the corresponding equation can be written as ${ }^{16}$ 


$$
\begin{aligned}
\frac{d p}{d t}= & {\left[p\left(\frac{\dot{m}_{u}}{\rho_{u}}+\frac{\dot{m}_{b}}{\rho_{b}}-\frac{d V}{d t}\right)+\zeta\left(\dot{Q}_{u}+Q_{b}+\dot{m}_{\mathrm{in}} h_{\mathrm{in}}+\dot{m}_{\mathrm{ex}} h_{\mathrm{ex}}\right.\right.} \\
& \left.\left.-\dot{m}_{u} h_{u}-\dot{m}_{b} h_{b}\right)\right] \frac{1}{[V(1-\zeta)]}
\end{aligned}
$$

with

$$
\zeta=\frac{V}{\frac{V_{u} c_{p, u}}{R_{u}}+\frac{V_{b} c_{p, b}}{R_{b}}} .
$$

Thus, except for combustion, Eqs. (1)-(3) are valid at any time including overlapping period. In the combustion process we consider two control volumes, for burned or unburned gases, separated by the propagating flame front, and as in other two-zone models ${ }^{17}$ equations giving the time evolution of $T_{u}, T_{b}$, and $p$ can be written as

$$
\begin{aligned}
\frac{d T_{u}}{d t}= & \frac{\dot{Q}_{u}+V_{u} \frac{d p}{d t}}{m_{u} c_{p, u}} \\
\frac{d T_{b}}{d t}= & \frac{\dot{Q}_{b}+\dot{m}_{b}\left(h_{u}-h_{b}\right)+V_{b} \frac{d p}{d t}}{m_{b} c_{p, b}} \\
\frac{d p}{d t}= & {\left[p\left(\frac{\dot{m}_{b}}{\rho_{b}}+\frac{\dot{m}_{u}}{\rho_{u}}-\frac{d V}{d t}\right)+\frac{\dot{Q}_{u} R_{u}}{c_{p, u}}\right.} \\
& \left.+\left(\dot{Q}_{b}+\dot{m}_{b}\left(h_{u}-h_{b}\right)\right) \frac{R_{b}}{c_{p, b}}\right]\left(V-\frac{V_{u} R_{u}}{c_{p, u}}-\frac{V_{b} R_{b}}{c_{p, b}}\right)^{-1} .
\end{aligned}
$$

Initial values for $T_{u}$ and $p$ at the beginning of combustion are given by Eqs. (1) and (2), whereas for $T_{b}$ initial value is calculated as the adiabatic flame temperature (at constant pressure). The different terms included in all the thermodynamic equations above require to be specified in the different steps of the system evolution accounting for the physical nature of the processes.

Our study will be only concerned with the stationary evolution of the cycle at a fixed angular velocity. Thus, we do not include in the set of differential equations, the mechanical equation for the relationship between the angular velocity and the forces on the piston. The friction forces will appear later on in the calculation of the work done by the engine. As a consequence, from the mechanical viewpoint we only need a relation between the volume of the chamber, $V$, and the crankshaft angle, $\varphi$. From the geometry of the system we obtain $^{15,22}$

$$
V=V_{0}+\frac{(r-1) V_{0}}{2}\left(\frac{1}{f}+1-\cos \varphi-\sqrt{\frac{1}{f^{2}}-\sin ^{2} \varphi}\right),
$$

where $V_{0}$ is the clearance volume, $f$ is the ratio of the crank radius, $a$, to the connecting rod length, $l$, and $r$ is the compression ratio. Volume time derivative is

$$
\frac{d V}{d t}=\frac{V_{0}}{2}(r-1) \psi \omega,
$$

where

$$
\psi=\sin \varphi+\frac{\sin \varphi \cos \varphi}{\sqrt{\frac{1}{f^{2}}-\sin ^{2} \varphi}} .
$$

\section{A. Gas flow rates}

The mass flow rate of a gas through a poppet valve is usually described by the equation for a compressible flow through a flow restriction, that is derived from a onedimensional isentropic flow analysis. ${ }^{15}$ For an ideal gas, flow rate depends on the upstream pressure, $p_{0}$, and temperature, $T_{0}$, the pressure just downstream the restriction, $p_{T}$ (assumed equal to the pressure at the restriction), and the orifice area, $A_{T}$, as

$$
\dot{m}=\frac{n_{v} C_{D} A_{T} p_{0}}{\sqrt{R T_{0}}}\left(\frac{p_{T}}{p_{0}}\right)^{\frac{1}{\gamma}}\left\{\frac{2 \gamma}{\gamma-1}\left[1-\left(\frac{p_{T}}{p_{0}}\right)^{\frac{\gamma-1}{\gamma}}\right]\right\}^{\frac{1}{2}},
$$

where $n_{v}$ refers to the number of intake/exhaust valves of the considered cylinder and $C_{D}$, that is determined experimentally, which is a discharge coefficient accounting for real gas flow effects. When $p_{T} / p_{0}=[2 / \gamma+1]^{\gamma / \gamma-1}$, the flow is choked or critical and flow rate is given by

$$
\dot{m}=\frac{n_{v} C_{D} A_{T} p_{0}}{\sqrt{R T_{0}}} \gamma^{\frac{1}{2}}\left(\frac{2}{\gamma+1}\right)^{\frac{\gamma+1}{2(\gamma-1)}} .
$$

The geometry of the valves for our simulation and the values of $A_{T}$ as a function of the valves lift are explained, for instance, in Ref. 24.

\section{B. Combustion}

As stated above, during the combustion period we consider two different control volumes, one for the unburned gases and the other for the burned ones and we make use of the quasidimensional model developed by $\mathrm{Keck}^{20}$ and extended by Beretta $e t$ al. ${ }^{19}$ The burning of the cylinder charge is considered as a turbulent flame propagation process. During this process, because of turbulences, unburned eddies of characteristic length $l_{t}$ are entrained into the flame zone at a velocity $u_{t}+S_{l}$, where $u_{t}$ is the characteristic speed (similar to the local turbulence intensity ${ }^{15}$ ) and $S_{l}$ the laminar flame speed. Then they are burned at velocity $S_{l}$ in a characteristic time, $\tau_{b}=l_{t} / S_{l}$. Thus combustion is described by solving this set of ordinary differential equations,

$$
\begin{aligned}
& \dot{m}_{e}=A_{f} \rho_{u}\left(u_{t}+S_{l}\right) \\
& \dot{m}_{b}=A_{f} \rho_{u} S_{l}+\frac{\left(m_{e}-m_{b}\right)}{\tau_{b}}
\end{aligned}
$$

where $m_{e}$ denotes the total mass inside the flame front, burned gas, and unburned eddies. $A_{f}$ is the area of the spherical flame front. During combustion these equations are coupled with the thermodynamic ones, Eqs. (4)-(6). Laminar burning speed is obtained from Güdler's model ${ }^{25}$ and $u_{t}, l_{t}$ 
from the empirical correlations built up by Keck. ${ }^{20} A_{f}$ is calculated from its radius, assuming an spherical flame front, and the radius from the volume of the gases inside the flame front, $V_{f}$, that following Bayraktar, ${ }^{17}$ can be expressed as

$$
V_{f}=V_{b}+\frac{m_{e}-m_{b}}{\rho_{u}}
$$

where $V_{b}=m_{b} / \rho_{b}$ is the volume of the burned gas mixture.

\section{Heat transfer}

In order to solve the thermodynamic set of differential equations it is necessary to assume a heat transfer model between the gas mixture inside the cylinder and the wall. Several models have been proposed in the literature for spark ignition four strokes engines. ${ }^{26,27}$ We shall take the formulation developed by Eichelberg, ${ }^{28}$ widely used in the literature, because its simplicity to calculate the instantaneous heat flow rate, $\dot{Q}_{s}$, in terms of a mean cylinder inner surface temperature, $T_{w}$, as

$$
\frac{\dot{Q}_{s}}{A_{s}}=2.43 v_{p}^{1 / 3}(p T)^{1 / 2}\left(T-T_{w}\right),
$$

where $A_{s}$ is the instantaneous surface transfer area, $p$ the instantaneous pressure inside the cylinder in bar, $T$ is the instantaneous bulk gas temperature, and $v_{p}=(2 / \pi) \omega a$ is the mean piston speed in terms of the crankshaft radius $a$ and the angular velocity $\omega$. Units of all variables except pressure are international system units.

\section{Engine friction}

To numerically evaluate the friction force affecting piston motion in the cylinder we assume that it depends linearly on the piston instantaneous speed, $\left|F_{\text {fric }}\right|=\mu|\dot{x}|$ where $\mu$ is an effective friction coefficient, and $x(t)$ the position of the piston respect to $\mathrm{BC}$. The effect of friction forces appears on the calculation of the net work as

$$
|W|=\left|W_{\text {gas }}\right|-\left|W_{\text {fric }}\right|
$$

where

$$
\begin{aligned}
& \left|W_{\text {gas }}\right|=\left|\int_{0}^{4 \pi} p\left(\frac{d V}{d \varphi}\right) d \varphi\right| \\
& \left|W_{\text {fric }}\right|=\frac{1}{A_{p}} \int_{0}^{4 \pi}\left|F_{\text {fric }}\right|\left|\frac{d V}{d \varphi}\right| d \varphi,
\end{aligned}
$$

and $A_{p}$ is the piston section.

\section{E. Working fluid}

In this study we elect as fuel iso-octane $\mathrm{C}_{8} \mathrm{H}_{18}$ (reference fuel for Otto's engines ${ }^{29}$ ) and the following chemical reaction:

$$
\begin{gathered}
\left(1-y_{r}\right)\left[\mathrm{C}_{8} \mathrm{H}_{18}+\alpha\left(\mathrm{O}_{2}+3.773 \mathrm{~N}_{2}\right)\right]+y_{r}\left[\beta_{r} \mathrm{CO}_{2}+\gamma_{r} \mathrm{H}_{2} \mathrm{O}\right. \\
\left.+\mu_{r} \mathrm{~N}_{2}+\nu_{r} \mathrm{O}_{2}+\varepsilon_{r} \mathrm{CO}+\delta_{r} \mathrm{H}_{2}\right] \rightarrow \beta \mathrm{CO}_{2}+\gamma \mathrm{H}_{2} \mathrm{O} \\
\quad+\mu \mathrm{N}_{2}+\nu \mathrm{O}_{2}+\varepsilon \mathrm{CO}+\delta \mathrm{H}_{2}
\end{gathered}
$$

TABLE I. Summary of the geometrical characteristics of the system employed in the validation of the simulation. Details on the geometry of the valves can be found in Ref. 15.

$a$, crank radius
$n_{v}$, number of pairs of valves
$l$, connecting rod length
$V_{0}$, clearance volume
$r$, compression ratio
$B$, bore

$44.45 \times 10^{-3} \mathrm{~m}$

1

$147 \times 10^{-3} \mathrm{~m}$

$1.05 \times 10^{-4} \mathrm{~m}^{3}$

7.86

$101.6 \times 10^{-3} \mathrm{~m}$ where the subscript $r$ refers to residual and $y_{r}$ denotes the molar fraction of exhaust gases in the intake process. We make use of the program CEA developed by NASA (Ref. 30) to solve combustion and calculate exhaust composition. We assume the unburned gas mixture as formed by the fuel, air, and nonreactive exhaust gases. Exhaust chemical composition appears directly as calculated from solving combustion.

Thermodynamic properties of all species are built as ideal gases and obtained from the constant pressure specific heat capacity, $c_{p, i}$, that is expressed as the following temperature depending polynomial: ${ }^{30}$

$$
\frac{c_{p, i}}{R}=a_{i 1} T^{-2}+a_{i 2} T^{-1}+a_{i 3}+a_{i 4} T+a_{i 5} T^{2}+a_{i 6} T^{3}+a_{i 7} T^{4}
$$

\section{F. Validating the simulation}

We have developed a computer code to solve the set of thermodynamical differential equations of the system and the combustion scheme previously considered by means of a fourth-order Runge-Kutta algorithm. The following simplifying hypothesis were assumed: i) The thermodynamical properties of each subsystem are homogeneous, ii) during compression and expansion system mass remains invariable, iii) there is no recirculation of exhaust gases, and iv) the only considered chemical reaction is that of combustion.

To briefly present the validation of our simulation scheme we have intended to reproduce the experimental results obtained by Beretta et al. ${ }^{19}$ by taking the geometry of their experimental cylinder and valves. A summary of the geometrical characteristics of the system is contained in Table I. Table II summarizes some configuration parameters of the simulation. Note that we take an average value ${ }^{15}$ for the discharge coefficient, $\mathrm{C}_{D}$, and that combustion starts at a fixed spark angle, $\varphi_{0}$. We show in Fig. 1 the comparison of

TABLE II. Configuration parameters of the simulations.

\begin{tabular}{lc}
\hline \hline$\varphi_{0}$, spark angle & $\frac{11}{6} \pi$ \\
$\mu$, friction coefficient & $16.0 \mathrm{~kg} / \mathrm{s}$ \\
$C_{D}$, discharge coefficient & 0.6 \\
$p_{\text {in }}$, intake pressure & $0.72 \times 10^{5} \mathrm{~Pa}$ \\
$T_{\text {in }}$, intake temperature & $350.0 \mathrm{~K}$ \\
$p_{\text {ex }}$, exhaust pressure & $1.05 \times 10^{5} \mathrm{~Pa}$ \\
$T_{\text {ex }}$, exhaust temperature & $600 \mathrm{~K}$ \\
$T_{w}$, cylinder internal wall temperature & $600 \mathrm{~K}$ \\
$\phi$, fuel ratio & 0.99 \\
\hline \hline
\end{tabular}




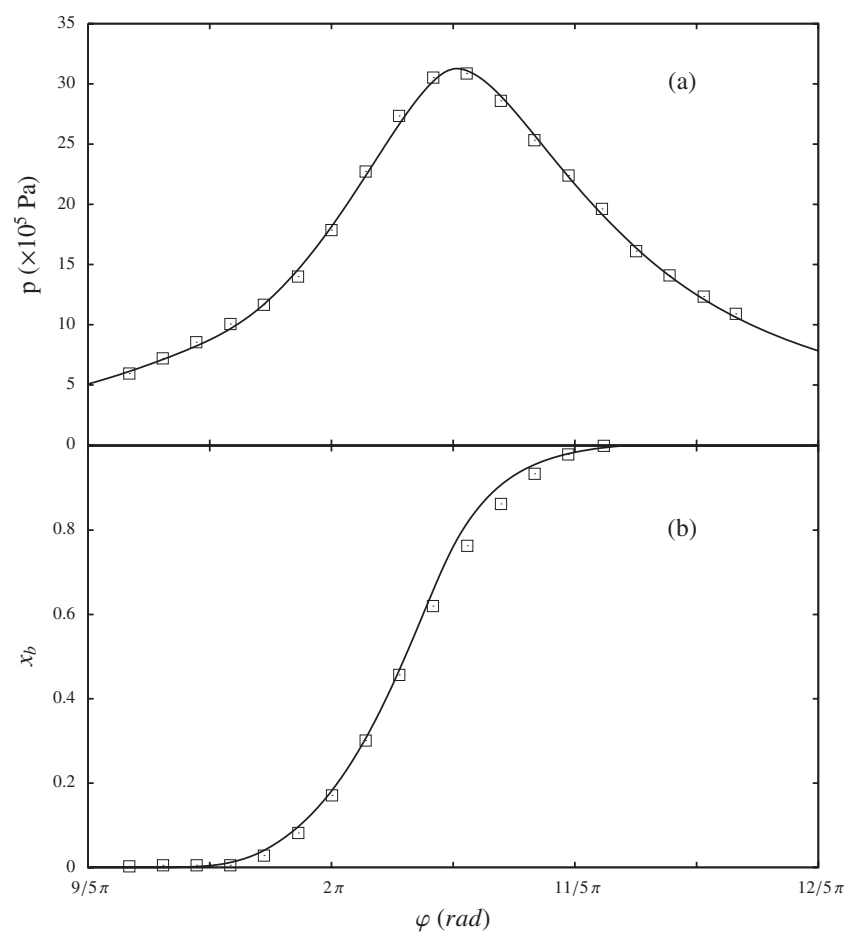

FIG. 1. (a) Pressure vs crankshaft angle and (b), evolution of the mass fraction of burned gases during combustion: comparison between the experimental results by Beretta et al. (Ref. 19) (๑) and our numerical results (solid line).

our simulation with the experimental ones for two key variables as a function of the crank angle. The agreement between the simulated and experimental pressures and the evolution of the burned mass gas fraction, $x_{b}$, are satisfactory. This essentially means that the combustion model we elect is adequate to account for both power output and efficiency.

\section{FINITE-TIME-THERMODYNAMICS MODEL}

The objective of working within the frame of FTT is to propose a simple analytical model to obtain the performance of the engine taking into account the main sources of irreversibility in the involved processes. Simulations developed in Sec. II shall allow us a precise checking application of this analytical model and the comparison between both approaches will provide a clear physical interpretation of the basic parameters that appear in FTT.

Our FTT irreversible model starts from the well-known reversible Otto cycle model (see Fig. 2 for notation) formed by two isochoric processes, combustion $(2 \rightarrow 3)$ and cooling $(4 \rightarrow 1)$. Compression $(1 \rightarrow 2)$ and power strokes $(3 \rightarrow 4)$ are considered adiabatic. Intake and exhaust processes are supposed isobaric, and so, do not influence the calculation of power or efficiency. Geometrically, the cycle can be characterized by a compression ratio, $r=V_{1} / V_{2}=V_{4} / V_{3} \geq 1$ and the ratio between the minimum and maximum temperatures, $\tau$ $=T_{1} / T_{3} \leq 1$. As usual, we consider three main irreversibility sources: any kind of internal irreversibilities associated with the working fluid (fluid frictions, viscosity, combustion, etc.) that are directly dissipated to the surroundings, ${ }^{5}$ friction work associated with piston dynamics, and heat transfers from the

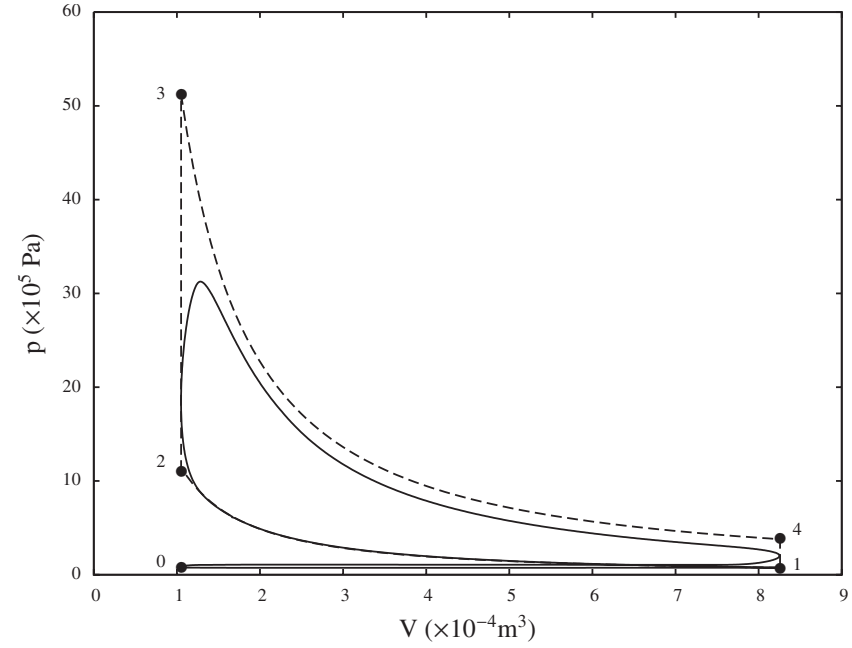

FIG. 2. $p$ - $V$ diagram of the cycle calculated from simulations (solid line) with the geometrical parameters of Beretta et al. at $\omega=109 \mathrm{rad} / \mathrm{s}$. It also included an idealized run of the simulation (dashed line) with isochoric combustion and without heat transfer and friction work losses.

working fluid to the surroundings through the cylinder walls. Next we model these contributions in simple analytical terms.

\section{A. Internal irreversibilities}

A usual way to include internal irreversibilities in the evaluation of the power output of the cycle is based on the idea by Özkaynak et $a l^{31,32}$ and $\mathrm{Chen}^{33}$ for Carnot-like cycles. If we denote $Q_{C}$ the heat release to the cold reservoir at temperature $T_{C}$ and $Q_{H}$ the heat absorbed from the hot at temperature $T_{H}$, we can write the Clausius inequality as an equality by introducing a term $I_{R}>1$ in such a way that

$$
I_{R} \frac{\left|Q_{H}\right|}{T_{H}}-\frac{\left|Q_{C}\right|}{T_{C}}=0
$$

with,

$$
\eta=1-I_{R} \frac{T_{C}}{T_{H}}=1-I_{R}\left(\frac{\left|Q_{C}\right|}{\left|Q_{H}\right|}\right)_{\text {rev }}=\frac{\left|W_{I}\right|}{\left|Q_{H}\right|_{\text {rev }}},
$$

where the subscript rev refers to reversible conditions. The output work including internal irreversibilities can be written as $\left|W_{I}\right|=\left|Q_{H}\right|_{\text {rev }}-I_{R}\left|Q_{C}\right|_{\text {rev }}$. Although in Otto models heat transfers do not happen in isothermal conditions, this formulation has been widely employed in the literature for these models taking always $I_{R}$ as a phenomenological and constant parameter. Angulo-Brown et al. ${ }^{34}$ argued that for a nonendoreversible Otto cycle, $I_{R}$ could be interpreted as the ratio between constant volume heat capacities of the combustion products and reactants, $I_{R}=C_{v, b} / C_{v, u}$, since heat absorption and release takes place in isochoric conditions. We shall see in Secs. IV-VI that our two-zone numerical simulation allows us to obtain precise values of $I_{R}$ for each working regime, i.e., as a $\omega$ function, and also a clear physical interpretation of its origin.

In our notation, $\left|Q_{H}\right|_{\text {rev }}=\left|Q_{23}\right|$ and $\left|Q_{C}\right|_{\text {rev }}=\left|Q_{41}\right|$, so $\left|W_{\text {rev }}\right|=\left|Q_{23}\right|-\left|Q_{41}\right|$ and 


$$
\left|W_{I}\right|=T_{3}\left[\bar{C}_{v, 23}\left(1-\tau r^{\bar{\gamma}_{u, 12}-1}\right)-I_{R} \bar{C}_{v, 41}\left(r^{\bar{\gamma}_{b, 34^{-1}}}-\tau\right)\right],
$$

where heat capacities and adiabatic coefficients are in general calculated as averages over the considered temperature interval. For instance, taking into account the chemical composition of the gas mixture in the cylinder before $(u)$ and after $(b)$ combustion,

$$
\begin{gathered}
\bar{C}_{v, 23}=\frac{1}{2}\left[C_{v, u}\left(T_{2}\right)+C_{v, b}\left(T_{3}\right)\right] ; \\
\bar{C}_{v, 41}=\frac{1}{2}\left[C_{v, b}\left(T_{4}\right)+C_{v, u}\left(T_{1}\right)\right]
\end{gathered}
$$

and

$$
\bar{\gamma}_{12}=\frac{\bar{C}_{p, 12}}{\bar{C}_{v, 12}}=\frac{C_{p, u}\left(T_{1}\right)+C_{p, u}\left(T_{2}\right)}{C_{v, u}\left(T_{1}\right)+C_{v, u}\left(T_{2}\right)} .
$$

The same kind of averages applies for other processes.

\section{B. Engine friction irreversibilities}

To evaluate the friction work associated with the piston motion inside the cylinder, we consider a friction force proportional to the instantaneous velocity in the same manner as that in the computer simulation model (Sec. II D). The velocity can be written in terms of the crank radius, $a$, the crank angle, $\varphi$, and the angular velocity, $\omega$, through the function $\psi$ defined in Eq. (9)

$$
|\dot{x}|=a \omega \psi \text {. }
$$

Thus, the whole friction work is calculated from Eq. (17). Considering that

$$
\frac{d V}{d \varphi}=\frac{V_{0}}{2}(r-1) \psi,
$$

we finally get

$$
\left|W_{\text {fric }}\right|=\frac{a \mu \omega V_{0}(r-1)}{2 A_{p}} \int_{0}^{4 \pi} \psi^{2} d \varphi .
$$

Note that this procedure is slightly different and more general (incorporates cylinder geometry) than the usual one in FTT (Refs. 12, 35, and 36) where the power losses due to friction are taken as $P_{\mu}=\mu v_{p}^{2}$, with the mean piston speed $v_{p}=\left|x_{4}-x_{3}\right| / t_{34}$ calculated from the extreme piston positions, $x_{3}$ and $x_{4}$, and the duration of the power stroke, $t_{34}$, considered as a fraction of the whole duration of the cycle.

\section{Irreversibilities associated with heat transfer}

The heat transfer rate from the working fluid to the engine surroundings through the cylinder walls, $\dot{Q}_{l}$, can be modeled in an easy way by assuming a convective heat transfer, $^{5}$

$$
\dot{Q}_{l}=\pi B h\left(\frac{B}{2}+x\right)\left(T-T_{w}\right),
$$

where $T$ is the instantaneous temperature of the working fluid inside the cylinder, $B$ is the cylinder bore diameter, $h$ is the heat transfer coefficient, and $T_{w}$ is the wall temperature. This simple procedure allow us to estimate losses associated with heat transfer in terms of a reduced set of parameters, including the time duration of the cycle.

In particular, assuming that the main heat transfer takes place in the power stroke and taking average values for piston position, $\bar{x}_{34}$, and fluid temperature, $\bar{T}_{34}$, it is obtained that ${ }^{5}$

$$
\bar{Q}_{l} \simeq \pi h B\left(\frac{B}{2}+\bar{x}_{34}\right)\left(\bar{T}_{34}-T_{w}\right) t_{34},
$$

where $t_{34}$ is the time duration of the power stroke. The lost work associated with the heat transfer can be written simply as $W_{Q}=\varepsilon \bar{Q}_{l}$ where $\varepsilon$ is a factor introduced by Mozurkewich and Berry ${ }^{5}$ in order to quantify the lost work. It was usually taken as a phenomenological constant parameter with value around $10 \%$. In Secs. IV and V we shall explicitly calculate this coefficient by comparing the FTT formulation with our computer simulation. Finally, assuming that $t_{34}$ is 0.25 times the whole duration of the cycle, $t,\left|W_{Q}\right|$ is given ${ }^{5}$ by

$$
\left|W_{Q}\right|=\frac{\pi \varepsilon h B t T_{3}}{16}\left[B+\frac{V_{0}}{A_{p}}(1+r)\right]\left(1+r^{1-\gamma}-2 \frac{T_{w}}{T_{3}}\right) .
$$

Thus, after giving simple models for all the considered irreversibility sources, the FTT approach allows to obtain the net work in the cycle as

$$
|W|=\left|W_{I}\right|-\left|W_{\text {fric }}\right|-\left|W_{Q}\right|,
$$

and the cycle efficiency as $\eta=|W| /\left|Q_{23}\right|$, with

$$
\left|Q_{23}\right|=\bar{C}_{v, 23} T_{3}\left(1-\tau r^{\bar{\gamma}_{u, 12}-1}\right) .
$$

Power is obtained as $P=|W| / t$ where $t$ is the cycle duration. Before applying this theoretical model to a particular configuration, it should be explained how to deal with the combustion temperature. This is the aim of the following subsection.

\section{Combustion temperature}

In previous FTT models the temperature after combustion, $T_{3}$, as well as the inlet temperature, $T_{1}$, were considered as fixed input parameters ${ }^{10,37}$ or calculated by taking constant temperature rates in heating and cooling branches. ${ }^{13,34,35}$ Nevertheless, in this paper $T_{3}$ is considered as the adiabatic flame temperature in the following manner: $T_{2}$ is obtained from the parameter $T_{1}$ (the only input temperature datum of the cycle) by considering the adiabatic equation in the 1-2 path, $T_{2}=T_{1} r^{\bar{\gamma}_{u, 12^{-1}}}$; afterwards $T_{3}$ is estimated by numerically solving the equation

$$
0=U_{P}\left(T_{3}\right)-U_{R}\left(T_{2}\right)
$$

where $U_{P}(T)$ and $U_{R}(T)$ are, respectively, the tabulated internal energy (with chemical term included) of products and reactants at those temperatures. This means that all internal energy, chemical and sensible, in reactants is transferred to products. Angulo-Brown et al. ${ }^{12}$ used the same procedure to calculate $T_{3}$. 
TABLE III. Summary of the geometrical and thermodynamical parameters used both in the theoretical results and in the simulated ones. $r$, compression ratio

$B$, bore

$T_{w}$, cylinder internal wall temperature

$T_{1}$, inlet temperature (FTT)

$h$, heat transfer coefficient (FTT)
$V_{0}$, clearance volume

$m$, mass of the gas mixture inside the cylinder (FTT)
10

$79.5 \times 10^{-3} \mathrm{~m}$

$49.639 \times 10^{-6} \mathrm{~m}^{3}$

$600 \mathrm{~K}$

$333 \mathrm{~K}$

$1305 \mathrm{~W} / \mathrm{m}^{2} \mathrm{~K}$

$4.176 \times 10^{-4} \mathrm{~kg}$

\section{NUMERICAL RESULTS}

\section{A. Theoretical model}

In order to briefly present the numerical performance of the cycle from the FTT model we assume a simple chemical reaction for iso-octane, the same that considered in Sec. II E but without taking into account the presence of exhaust residues within the reactants. Note that this reaction is simpler than that considered in the simulations because combustion products are not taken as reactants. We elect this reaction in order to simplify as much as possible FTT scheme, after proving that this not affect the results shown in this section.

Although the theoretical scheme allows for the consideration of temperature dependent heat capacities, we take here a constant value for $c_{v}=5 R / 2$ and the corresponding enthalpy in order to keep the simplicity of the method. We also consider constant $(\omega$-independent) irreversibility parameters, with values taken from the literature, $I_{R}=1.4,{ }^{34,37} \varepsilon=0.1$, and $\mu=16 \mathrm{~kg} / \mathrm{s}$ (Ref. 15) (which corresponds to an average friction force of $100 \mathrm{~N}$ ). With respect to geometrical parameters of the cylinder and thermodynamic input data, we consider the numerical values contained in Table III. ${ }^{19}$

The evolution of power and efficiency with the crankshaft rotational speed, $\omega$, are shown in Fig. 3(a). Both curves show that theoretical computations give good orders of magnitude for maximum power and efficiency and a paraboliclike behavior with different maxima positions for each one. This is in qualitative concordance with other theoretical and numerical computations ${ }^{34,37}$ and shows that a simple FTT model with constant irreversibility parameters is capable to reproduce the loop-shaped power-efficiency curves shown by real devices (see Fig. 4). Moreover, the rotational velocity giving maximum power is greater than that of the maximum efficiency, thus when we move along the $P-\eta$ curve by increasing $\omega$ we do it counterclockwise. This behavior is not sensitive to the election of slightly different irreversibility parameters in the FTT model.

From this model it is also possible to study the evolution of work losses associated with the different irreversibility sources with the working regime of the engine. In order to understand those differences we have calculated separately the different terms that contribute to the net work in this way:

$$
\begin{aligned}
|W| & =\left|W_{\text {rev }}\right|-\left(\left|W_{\text {rev }}\right|-\left|W_{I}\right|\right)-\left|W_{\text {fric }}\right|-\left|W_{Q}\right| \\
& \equiv\left|W_{\text {rev }}\right|-\left|\sum W_{l}\right| .
\end{aligned}
$$

In Fig. 5(a) we plot the difference between the work obtained

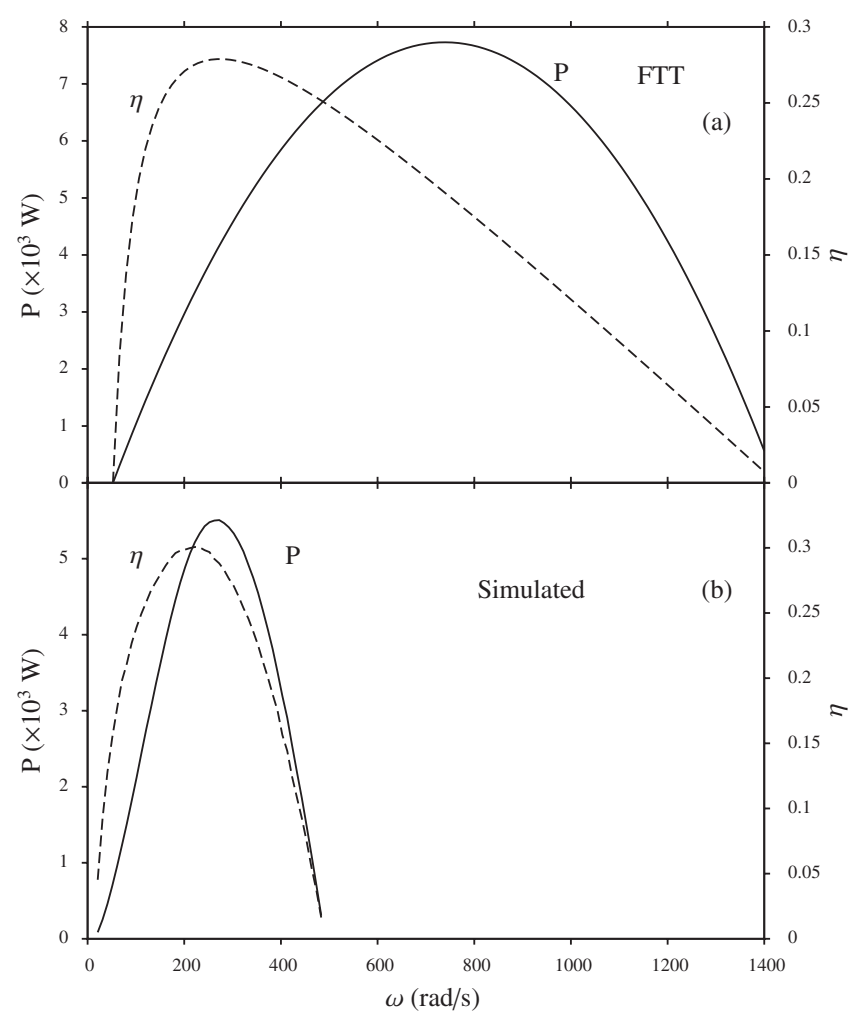

FIG. 3. Evolution of the power output and efficiency with $\omega$ : (a) for the theoretical model, taking $I_{R}=1.4$ and $\varepsilon=0.1$ as phenomenological constant parameters (other parameters considered are those in Table III) and (b), from the simulations as performed in Sec. IVB.

under reversible conditions, $\left|W_{\text {rev }}\right|$, and the work obtained by considering internal irreversibilities, $\left|W_{I}\right|$. The difference is constant because we considered $I_{R}$ and $T_{3}$ (and thus $\tau$ ) independent of $\omega$. The lost work associated with engine frictions is, of course, linear with $\omega$ and the lost work associated with heat transfer from the cylinder walls to the surroundings, $\left|W_{Q}\right|$, decreases monotonically. As a consequence the net lost work presents a minimum around $270 \mathrm{rad} / \mathrm{s}$. Comparing relative importance, at low $\omega$, heat transfer irreversibility, $\left|W_{Q}\right|$,

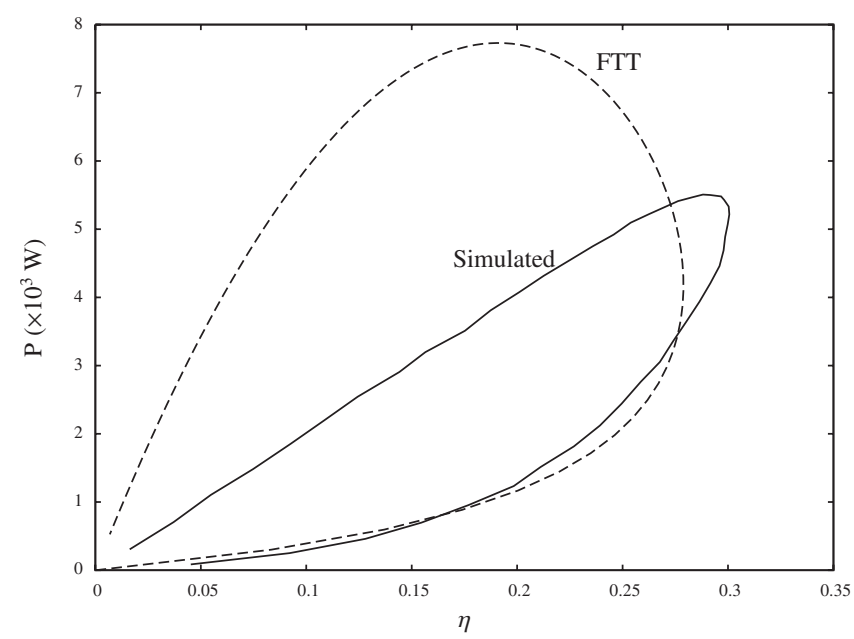

FIG. 4. $P-\eta$ curves from the theoretical model with constant irreversibility parameters (see Sec. IV A) (dashed line) and the simulations (see Sec. IVB) (solid) obtained by eliminating the rotational speed $\omega$. 


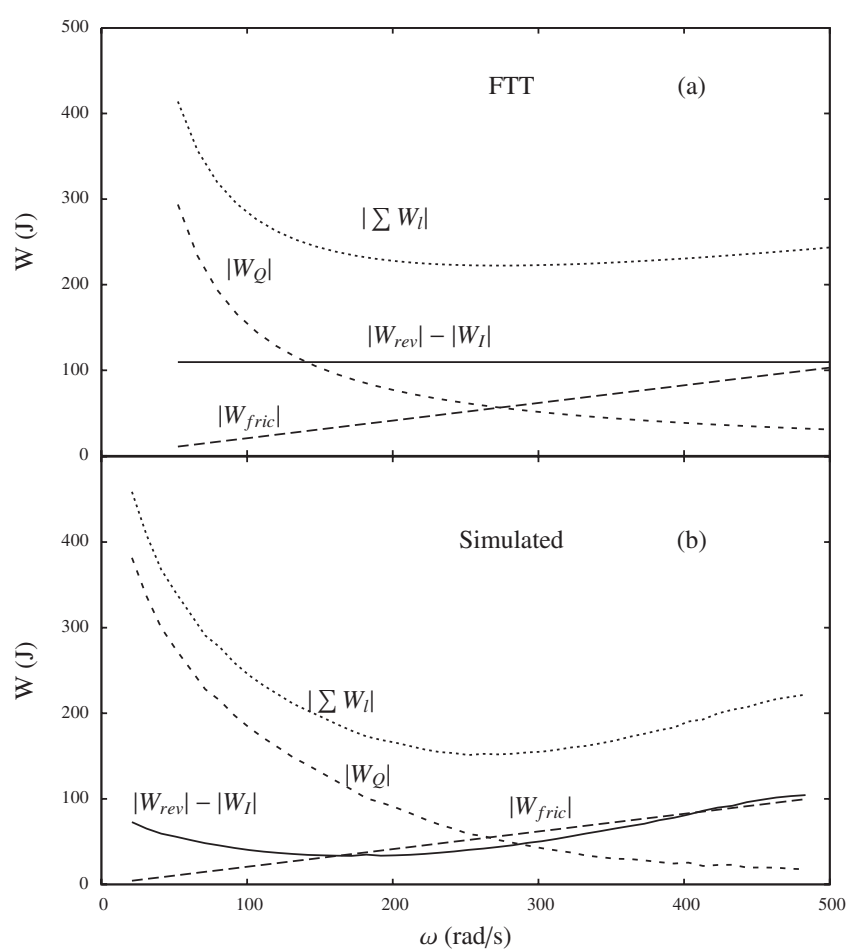

FIG. 5. Different contributions to the total lost work, $\left|\sum W_{l}\right|=\left(\left|W_{\text {rev }}\right|-\left|W_{I}\right|\right)$ $-\left|W_{\text {fric }}\right|-\left|W_{Q}\right|$ (a) as obtained from the theoretical model and (b) as obtained from the simulations.

is over the rest of losses. At any $\omega,\left|W_{\text {rev }}\right|-\left|W_{I}\right|$ is greater than $\left|W_{\text {fric }}\right|$ but the difference decreases as $\omega$ increases.

\section{B. Numerical results from computer simulations}

In order to simulate a more elaborated engine than that of the validation of Sec. II F, we consider here a four valve cylinder $\left(n_{v}=2\right)$. All the required parameters for the simulation not included in Table III are considered as in Sec. II F, except the valves geometry that are taken with standard dimensions. ${ }^{15}$ In order to establish the border conditions the outer pressure is calculated through the ideal gas law from cylinder volume, considering the FTT values of mass, $m$, and the inlet temperature, $T_{1}$, (see Table III). Afterwards, due to the nonideality of the cycle, the intake mass changes with $\omega$.

Before presenting the results of the simulations we should note that in order to evaluate the thermal efficiency we have calculated the heat input on the following manner. We consider $T_{1}$ as the temperature of the mixture in the simulated cylinder when the piston reaches BC. The compression stroke is considered as adiabatic and thus $T_{2}$ is calculated. $T_{3}$ is considered as an adiabatic flame temperature, Eq. (33). From the values of these temperatures we obtain $\tau$ and $\left|Q_{23}\right|$ using Eq. (32). $\bar{C}_{v, 23}$ and $\bar{\gamma}_{u, 12}$ are calculated as averages on the temperature of the corresponding process. Then, we calculate thermal efficiency, $\eta=|W| /\left|Q_{23}\right|$, from the simulations with a similar heat input that in the theoretical model. An alternative way to calculate heat input in the simulations is by using the calorific power of the fuel, ${ }^{15}$ but this would mean the election of different input energy origins in simulations and theoretical calculations. Power is obtained as $P=|W| \omega /(4 \pi)$ where $|W|$ is calculated from Eq. (15).
Simulations predict power and efficiency curves with a maximum in terms of the rotational speed $\omega$ [see Fig. 3(b)] and the existence of loop shaped $P$ - $\eta$ graphs (see Fig. 4), but quantitative differences between theoretical and simulated results are obvious. Moreover, the $\omega$ interval in which power is positive is much wider in FTT. This is because in the theoretical calculations the mass of the mixture is independent of $\omega$, while in simulations the total mass decrease with increasing $\omega$ and so does the power output. We shall go back to this point in Sec. V.

We have also calculated from the simulations the different contributions to the lost work [Eq. (34)]. $\left|W_{\text {rev }}\right|$ is calculated from a simulation without heat transfer to the cylinder walls, with null friction and taking $T_{3}$ as the adiabatic flame temperature. $\left|W_{I}\right|$ is considered as the net work obtained from the simulations when friction forces and heat transfer to the cylinder wall are neglected. $\left|W_{\text {fric }}\right|$ is calculated directly from Eq. (17) and $\left|W_{Q}\right|=\left|W_{I}\right|-\left|W_{\text {fric }}\right|-|W|$. $\left|\Sigma W_{l}\right|$ represents the addition of all work losses. All these terms are depicted in Fig. 5(b). From comparison with plots in Fig. 5(a) we mention three valuable consequences: i) As $\omega$ increases, losses associated with internal irreversibilities in the simulations are no longer constant but they have a minimum around 190 $\mathrm{rad} / \mathrm{s}$; ii) the evolution of $\left|W_{Q}\right|$ and $\left|W_{\text {fric }}\right|$ is similar in both methods, $\left|W_{\text {fric }}\right|$ increases linearly with $\omega$ and $\left|W_{Q}\right|$ decreases, more like an exponential with a single slope in simulations and with two clear different decays in FTT; and iii) the addition of work losses, $\left|\Sigma W_{l}\right|$, has a deeper minimum in simulations, suggesting that the consideration of an internal irrrevesibility factor, $I_{R}$, independent of $\omega$ in FTT is not a good approximation.

\section{SPEED DEPENDENT PARAMETERS FROM THE SIMULATIONS}

Our deal now is how to get more realistic FTT results, using the simulation as a benchmark for the calculation of $\omega$-dependent irreversibility parameters. In other words, the question is if we can maintain the basic ideas under the FTT model but calculate some of the involved parameters to precisely reproduce simulations. Additionally, this will give us a more physical insight of the parameters characterizing global irreversibilities.

\section{A. Temperatures and mass}

In the numerical application of our theoretical model, the inlet temperature $T_{1}$ was considered as a fixed parameter ${ }^{12,37}$ independent of the rotational velocity. The calculation of $T_{2}$ and $T_{3}$ was explained in Sec. IIID. Now, we shall consider an $\omega$-dependent $T_{1}$ taken from the idealized version of simulations. In that situation, $T_{1}$ is taken as the temperature when the piston is at BC before combustion for each $\omega$. Then $T_{3}$ is calculated from an adiabatic isochoric combustion and $T_{2}$ from the adiabatic equation of process 1-2.

The $\omega$ behavior for $T_{1}$ and $T_{3}$ are displayed in Fig. 6(a). Inlet temperature shows a monotonic increase with $\omega$ changing from 360 up to $430 \mathrm{~K}$ approximately, which represents roughly a variation at $20 \%$. On the contrary, temperature after combustion, presents a maximum of $3178 \mathrm{~K}$ at 210 


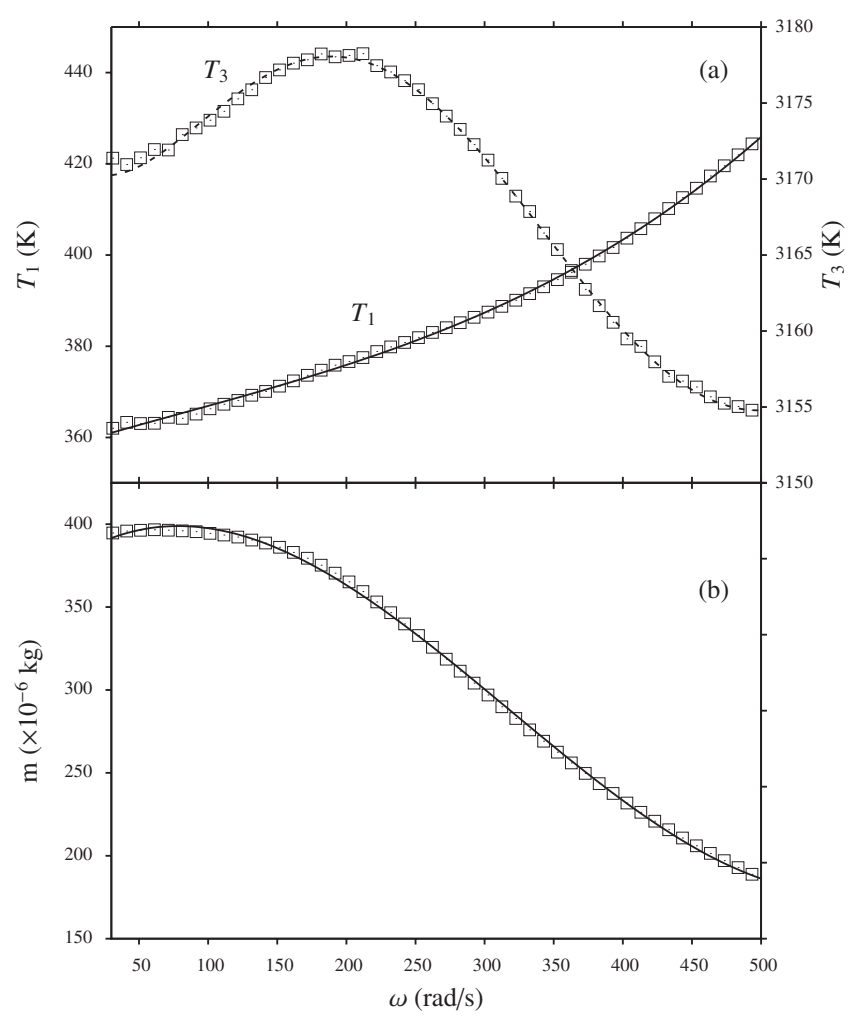

FIG. 6. (a) Dependence of the inlet temperature, $T_{1}$, and the maximum cycle temperature, $T_{3}$, of the so-called reversible simulation with $\omega$, and corresponding fitted polynomials. (b) Evolution of the mass contained in the cylinder with $\omega(\bullet)$ and the corresponding fitted polynomial (solid line) introduced in the theoretical FTT calculations of Sec. V.

$\mathrm{rad} / \mathrm{s}$ and then decreases to a minimum value of $3155 \mathrm{~K}$ at the highest $\omega$. This represents a difference of $7 \%$ between its minimum and maximum values.

Another characteristic parameter of the theoretical model that was considered as an input fixed parameter in previous works ${ }^{10,37}$ is the mass of the gas mixture inside the cylinder, $m$. Its influence on the output power and efficiency is basic because the energy entering the system is proportional to $m$. Its calculation from simulations is straightforward considering a simulation without heat and friction losses when inlet valve is closed. The evolution of $m$ for our cylinder configuration presents [see Fig. 6(b)] a maximum at low revolutions and a monotonic decay at larger $\omega$ in such a way that the maximum value represents nearly two times the mass at the highest velocity.

\section{B. Irreversibility parameters}

We now deal with the calculation of the irreversibililty parameters $I_{R}$ and $\varepsilon$ from the simulations as a function of the working regime of the engine. In order to obtain a simple expression to calculate the internal irreversiblity parameter, $I_{R}$, we express Eq. (22) as

$$
\left|W_{I}\right|=T_{3}\left(\zeta_{1}-I_{R} \zeta_{2}\right)
$$

where

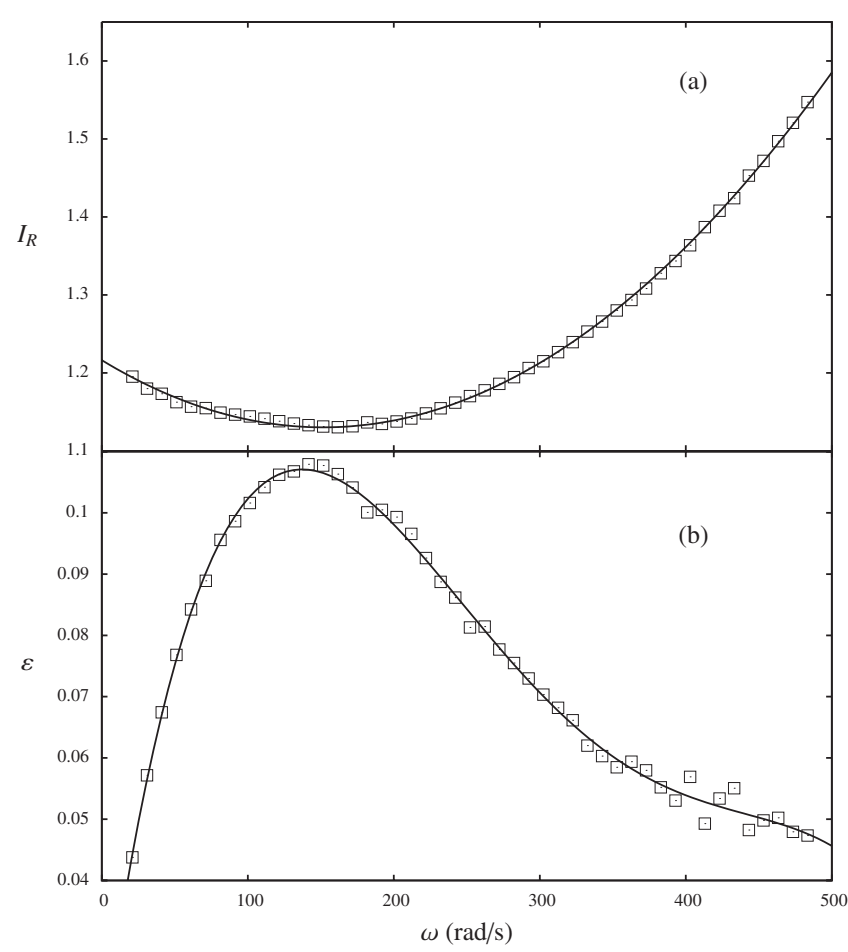

FIG. 7. (a) Internal irreversibility factor, $I_{R}$, as a function of $\omega$ obtained from the simulations and its corresponding fitted polynomial. (b) Irreversibility parameter, $\varepsilon$, associated with heat transfer through the cylinder walls as obtained from the simulations and its corresponding fitted polynomial.

$$
\begin{aligned}
& \zeta_{1}=\bar{C}_{v, 23}\left(1-\tau r^{\bar{\gamma}_{u, 12}-1}\right) \\
& \zeta_{2}=\bar{C}_{v, 41}\left(r^{\bar{\gamma}_{b, 34^{-1}}}-\tau\right) .
\end{aligned}
$$

Thus, it is possible to write $I_{R}$ as

$$
I_{R}=\frac{\zeta_{1}}{\zeta_{2}}-\frac{\left|W_{I}\right|}{\left|W_{\text {rev }}\right|} \frac{\zeta_{1}-\zeta_{2}}{\zeta_{2}}
$$

that will be $\omega$ dependent because temperatures and thus $\zeta_{1}$ and $\zeta_{2}$ are functions of the engine speed. As commented in Sec. IVB, $\left|W_{I}\right|$ is obtained from the simulations neither considering heat transfer work losses nor friction irreversibilities and furthermore $\left|W_{\text {rev }}\right|$ considering that the combustion process is adiabatic and isochoric. This means that the we assume as internal irreversibilities those coming from nonisochoric combustion, heat release processes during combustion and also from pumping the gas mixture in and out the cylinder. Figure 7(a) shows a paraboliclike behavior for $I_{R}$ that first decays as $\omega$ increases, presents a minimum, $I_{R}=1.15$, at $160 \mathrm{rad} / \mathrm{s}$ and then progressively increases with $\omega$ taking values greater that 1.5 . This means that there is a particular regime in which $I_{R}$ is minimum, while at higher velocities internal losses become quite more significant.

The factor $\varepsilon$ that relates heat transfer through the cylinder walls with work losses can be calculated by identifying Eq. (30) with $\left|W_{Q}\right|=\left|W_{I}\right|-\left|W_{\text {fric }}\right|-|W|$, where $\left|W_{I}\right|$ is computed as before. Thus, now it will be a function of $\omega$ because $T_{3}$ and the temperature ratio $\tau$ do. Figure 7(b) shows a parabolic shape for $\varepsilon$ with a maximum in the rotational velocity interval. The maximum is located at $\omega$ around $160 \mathrm{rad} / \mathrm{s}$ that is precisely the regime at which $I_{R}$ presents a minimum [Fig. 


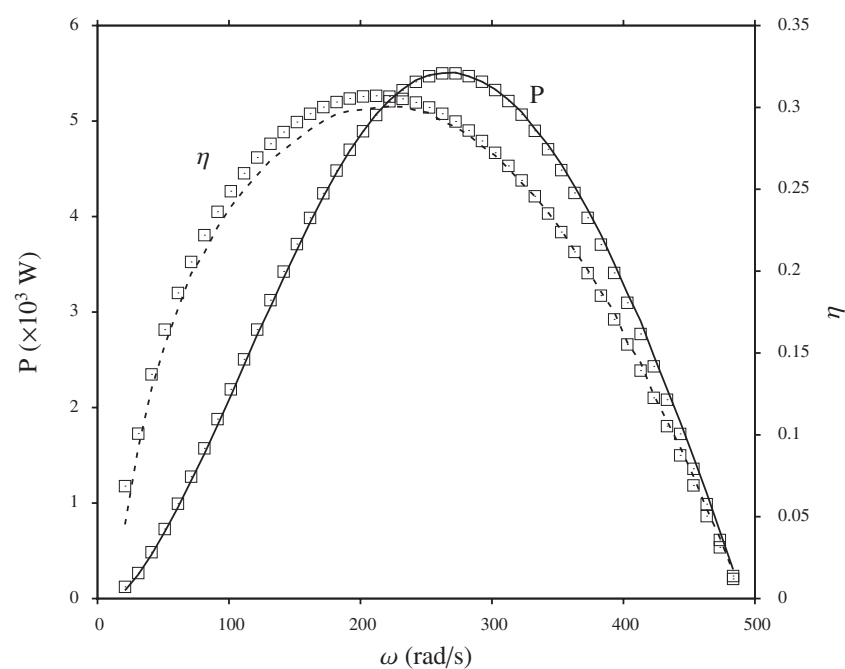

FIG. 8. Comparison of the evolution of the power output and efficiency with the rotational velocity as obtained from numerical simulations (lines) and from the theoretical calculations $(\cdot)$ considering fitted $\omega$-dependent polynomials.

$7(\mathrm{a})]$. The interval of values of $\varepsilon$ is quite wide, ranging from 0.04 at the extreme values of $\omega$ to almost 0.11 , its maximum value.

Numerical values of $I_{R}(\omega)$ and $\varepsilon(\omega)$ are similar to those found in the literature when considered as constant phenomenological parameters: integral averages over all $\omega$ range of $I_{R}(\omega)$ and $\varepsilon(\omega)$ give us values of $\bar{I}_{R}=1.23$ and $\bar{\varepsilon}=0.08$ for our cylinder. These values compare favorably with the values 1.4 and 0.1 we took in Sec. IV A from previous works. ${ }^{12,34,37}$

\section{Engine performance}

We have performed polynomial fits of temperatures, gas mixture mass, and irreversibility parameters $I_{R}$ and $\varepsilon$ as functions of $\omega$ and compared the results obtained from the complete simulations and the theoretical model incorporating those functions. Results for output power and efficiency are displayed in Fig. 8. Theoretical results for power compare very well with the simulated ones in all $\omega$ interval. The same applies for the thermodynamic efficiency, although a small difference is observed at $\omega$ near to the maximum efficiency region. Note that besides of the shape of the curves themselves, now the horizontal scales of theoretical and simulated results coincide. This is because of the consideration of the $m(\omega)$ inside the cylinder: as $\omega$ increases the mass, power and efficiency rapidly decrease, approaching to zero much faster than if mass is assumed constant. Also in the parametric $P-\eta$ curves plotted in Fig. 9(a) are observed the good results obtained from the theoretical model when the mentioned parameters are considered as $\omega$-dependent and estimated from simulations.

Of course, if we represent now the different contributions to the lost work from theory, we shall obtain a figure very similar to Fig. 5(b). The minimum of $I_{R}(\omega)$ provokes a minimum in $\left|W_{\text {rev }}\right|-\left|W_{I}\right|$ and the parabolic shape of $\varepsilon(\omega)$ provokes moreover an almost exponential decay of $\left|W_{Q}\right|$ as a function of $\omega$.

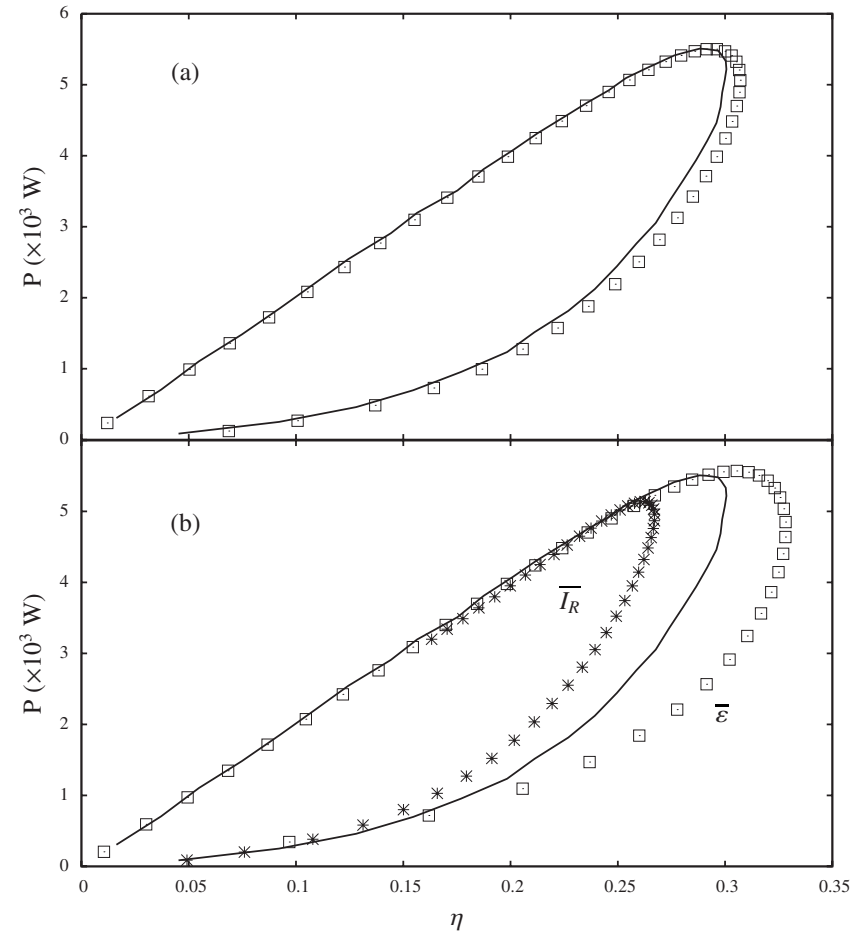

FIG. 9. (a) $P-\eta$ loops as obtained from numerical simulations (line) and from the theoretical calculations $(\square)$ considering fitted $\omega$-dependent polynomials by eliminating $\omega$. (b) Influence of average irreversibility parameters, $\bar{I}_{R}=1.23(*)$ or $\bar{\varepsilon}=0.08(\bullet)$, and comparison with simulation results (solid line). In the theoretical model, mass in the cylinder and temperatures are considered as fitted polynomials (Sec. V) and for each curve the other irreversibility parameter was taken as a function of $\omega$.

\section{DISCUSSION AND CONCLUSIONS}

In order to check the validity of our FTT model of a spark ignition engine, we have presented and validated a quasidimensional simulation of a piston-cylinder system undergoing an Otto cycle. The simulation considers a two-zone turbulent model for combustion and allows the consideration of several cylinder and valves geometry, reproducing realistic modern engines. The modularity of the simulation scheme allows to evaluate the essential contributions to work losses: frictions, heat transfer through the cylinder walls, and internal irreversibilities associated with combustion itself.

Moreover, we have developed a simple analytical thermodynamical model that as a first approximation gives interesting results when comparing results for the performance of the cycle with the simulated ones. Taking constant cylinder mass, constant inlet temperature, and phenomenological values for the parameters that globally characterize friction, heat transfer, and internal irreversibilities, the theoretical model reproduce the order of magnitude of power output and thermal efficiency as functions of the working regime, i.e., the rotational speed of the crankshaft, $\omega$. Nevertheless, the consideration of $\omega$-independent values for those variables do not allow to get the width of the $\omega$-interval giving positive output power, neither good numerical values for maximum power output or maximum efficiency. As a consequence, the observed loop-shaped $P-\eta$ curves are satisfactorily reproduced.

By means of simulations we are capable to obtain for 
each $\omega$ the mass of the gas mixture inside the cylinder, the main temperatures of the cycle and the irreversibility parameters associated with internal losses $\left(I_{R}\right)$ and heat transfer $(\varepsilon)$. Both irreversibility parameters show a parabolic behavior in the allowed $\omega$ range with a minimum for $I_{R}(\omega)$ and a maximum for $\varepsilon(\omega)$ at intermediate rotational speed (around $160 \mathrm{rad} / \mathrm{s}$ ). The inclusion of the corresponding polynomial fits in the theoretical scheme allows a precise reproduction of the results of the simulations: allowed $\omega$-interval and numerical values of maximum power and efficiency. Average values of the computed irreversibility parameters, $\bar{I}_{R}$ and $\bar{\varepsilon}$, compare quite well with other assumed as phenomenological in previous works. In any case we have checked [see Fig. 9(b)] that the consideration in the theoretical scheme of those average values is not enough to reproduce realistic numbers for maximum power and efficiency. This means that from our theoretical model, simulation results are only numerically reproduced considering explicitly the functions $T_{1}(\omega)$, $T_{3}(\omega), m(\omega), I_{R}(\omega)$, and $\varepsilon(\omega)$.

In summary, a simple analytical model based upon basic thermodynamic considerations explicitly including chemical reactions, with irreversibilities associated with friction, heat transfer, and internal losses is capable to reproduce somewhat sophisticated simulations of an spark ignition fourstroke cylinder engine. Comparison of the numerical results with theoretical and simulated cylinders gives us a physical insight of the global irreversibilities provoking power and efficiency losses and a way to quantify them. We hope that the results reported here could be a guide to improve theoretical models of internal combustion engines.

\section{ACKNOWLEDGMENTS}

A. M. and A. C. H. acknowledge financial support from Ministerio de Educación y Ciencia of Spain (Grant Nos. FIS2005-05081 FEDER and FIS2006-03764 FEDER) and Junta de Castilla y León (Grant No. SA054A08). P. L. C. -R. gratefully acknowledges a grant from Grupo SantanderUniversidad de Salamanca.

${ }^{1}$ S. Sieniutycz and P. Salamon, Finite-Time Thermodynamics and Thermoeconomics (Taylor \& Francis, London, 1990).

${ }^{2}$ A. Bejan and E. Mamut, Thermodynamic Optimization of Complex Energy Systems, NATO Science Series (Kluwer, Dordrecht, 1999).

${ }^{3}$ R. Berry, V. Kazakov, S. Sieniutycz, Z. Szwast, and A. Tsirlin, Thermo- dynamic Optimization of Finite-Time Processes (Wiley, New York, 2000). ${ }^{4} \mathrm{~L}$. Chen and F. Sun, Advances in Finite-Time Thermodynamics (Nova Science, Hauppauge, NY, 2004).

${ }^{5}$ M. Mozurkewich and R. Berry, J. Appl. Phys. 53, 34 (1982).

${ }^{6}$ B. M. Aizenbud, Y. B. Band, and O. Kafri, J. Appl. Phys. 53, 1277 (1982).

${ }^{7}$ L. Chen, C. Wu, F. Sun, and S. Cao, Energy Convers. Manage. 39, 643 (1998).

${ }^{8}$ V. Orlov and R. Berry, J. Appl. Phys. 74, 4317 (1993).

${ }^{9}$ F. Angulo-Brown, J. Fernández-Betanzos, and C. A. Pico, Eur. J. Phys. 15, 38 (1994).

${ }^{10}$ A. C. Hernández, A. Medina, J. Roco, and S. Velasco, Eur. J. Phys. 16, 73 (1995).

${ }^{11}$ S. A. Klein, ASME J. Eng. Gas Turbines Power 113, 511 (1991).

${ }^{12}$ F. Angulo-Brown, T. D. Navarrete-González, and J. A. Rocha-Martínez, in Recent Advances in Finite-Time Thermodynamics, edited by C. Wu, L. Chen, and J. Chen (Nova Science, Commack, NY, 1999).

${ }^{13}$ Y. Ge, L. Chen, F. Sun, and C. Wu, Int. J. Exergy 2, 274 (2005).

${ }^{14}$ O. Ozsoysal, Energy Convers. Manage. 47, 1051 (2006).

${ }^{15}$ J. B. Heywood, Internal Combustion Engine Fundamentals (McGrawHill, New York, 1988)

${ }^{16}$ C. Borgnakke, P. Puzinauskas, and Y. Xiao, University of Michigan, Technical Report No. UM-MEAM-86-35, 1986.

${ }^{17}$ H. Bayraktar and O. Durgun, Energy Sources 25, 439 (2003).

${ }^{18}$ C. Rakopoulos and E. Giakoumis, Prog. Energy Combust. Sci. 32, 2 (2006).

${ }^{19}$ G. P. Beretta, M. Rashidi, and J. C. Keck, Combust. Flame 52, 217 (1983).

${ }^{20}$ J. C. Keck, Proceedings of the 19th Symposium (International) on Combustion (Combustion Institute, Pittsburgh, 1982), pp. 1451-66.

${ }^{21}$ L. Guzzella and C. H. Onder, Introduction to Modeling and Control of Internal Combustion Engine Systems (Springer, New York, 2004).

${ }^{22} \mathrm{R}$. Stone, Introduction to Internal Combustion Engines (Macmillan, London, 1999).

${ }^{23}$ A. Fischer and K. H. Hoffmann, J. Non-Equilib. Thermodyn. 29, 9 (2004).

${ }^{24} \mathrm{~J}$. B. Heywood, Internal Combustion Engine Fundamentals (McGrawHill, New York, 1988), Chap. 6, pp. 220-230.

${ }^{25}$ O. L. Gülder, SAE Technical Report No. 841000, 1984.

${ }^{26} \mathrm{R}$. Stone, Introduction to Internal Combustion Engines (Macmillan, London, 1999), Chap. 10, pp. 429-433.

${ }^{27}$ M. I. Karamangil, O. Kaynakli, and A. Surmen, Energy Convers. Manage. 47, 800 (2006)

${ }^{28}$ G. Eichelberg, Engineering (London) 149 (1939).

${ }^{29} \mathrm{C}$. R. Ferguson, Internal Combustion Engines: Applied Thermosciences (Wiley, New York, 1986).

${ }^{30}$ B. J. McBride and G. Sanford, Users Manual 1311, National Aeronautics and Space Administration, NASA, 1996 http://www.grc.nasa.gov/WWW/ CEAWeb/

${ }^{31}$ S. Özkaynak, S. Götkun, and H. Yavuz, J. Phys. D 27, 1139 (1994).

${ }^{32}$ S. Özkaynak, J. Phys. D 28, 2024 (1995).

${ }^{33}$ J. Chen, J. Phys. D 27, 1144 (1994).

${ }^{34}$ F. Angulo-Brown, J. A. Rocha-Martínez, and T. D. Navarrete-González, J. Phys. D 29, 80 (1996).

${ }^{35}$ X. Qin, L. Chen, F. Sun, and C. Wu, Eur. J. Phys. 24, 359 (2003).

${ }^{36}$ Y. Ge, L. Chen, F. Sun, and C. Wu, Appl. Energy 81, 397 (2005).

${ }^{37}$ J. M. M. Roco, A. Medina, A. Calvo Hernández, and S. Velasco, Revista Española de Física 12, 39 (1998) [in Spanish]. 